\title{
Preparation and Characterization of Different Shapes of Silver Nanostruc- tures in Aqueous Solution
}

\author{
Zusing Yang ${ }^{1}$, Tai-Chia Chiu ${ }^{1}$ and Huan-Tsung Chang ${ }^{*}, 1,2$ \\ ${ }^{I}$ Department of Chemistry, National Taiwan University, Taipei, Taiwan \\ ${ }^{2}$ Department of Natural Science Education, National Taitung University, Taitung, Taiwan
}

\begin{abstract}
We have developed a new and simple one-pot synthetic approach for the preparation of single-crystalline rose-, spike-, and snowflake-shaped silver nanostructures (NSs) in aqueous solution. We obtained these variously shaped Ag NSs by carefully controlling the nature and concentration of the stabilizers, which included sodium acetate, sodium citrate, and poly(ethylene glycol). To the best of our knowledge, this paper is the first to describe the preparation of roseshaped Ag NSs, also called Ag nanoflowers (NFs), through such a simple synthetic route. The thus-prepared Ag NFs exhibited a specific surface area of $15.6 \mathrm{~m}^{2} \mathrm{~g}^{-1}$, a contact angle of $97.6 \pm 2.2^{\circ}$, high conductivity $\left(1.97 \times 10^{6} \mathrm{~S} / \mathrm{cm}\right)$, and efficient optothermal conversion efficiency (the temperature rose $23.03 \pm 0.21{ }^{\circ} \mathrm{C}$ after laser irradiation at $808 \mathrm{~nm}$ for $3 \mathrm{~min}$ ). The snowflake-shaped Ag NSs allowed the enhancement of Raman scattering (SERS) signals of rhodamine 6G by a factor of $2.4 \times 10^{6}$, leading to a detection limit of $10 \mathrm{nM}$.
\end{abstract}

\section{INTRODUCTION}

It is great interest in preparation of nanostructures (NSs) in various morphologies because of the shape- and sizedependence of optical and electronic properties [1-8]. Flower-shaped NSs exhibiting properties that differ from those of their corresponding bulk materials are among the most interesting types of NSs [9-11]. For example, it has been demonstrated that (a) 50-nm-diameter $\mathrm{MgO}$ nanoflowers (NFs) possess a relatively higher dielectric constant than do $\mathrm{MgO}$ micropowders in the frequency ranging from $50 \mathrm{~Hz}$ to $5 \mathrm{MHz}$ [9], (b) GaP NFs provide a greater cathode luminescence than do $\mathrm{GaP}$ nanowires [10], and (c) $\mathrm{SnO}_{2} \mathrm{NFs}$ exhibit super-hydrophobic properties [11]. Recently, large flower-like $\mathrm{TiO}_{2} \mathrm{NFs}$ with 250 400 nm in diameter have also been applied in dye-sensitized solar cells as scattering layers to achieve a high efficiency of energy conversion [12]. $\mathrm{TiO}_{2} \mathrm{NFs}$ have been demonstrated useful for photodegradation of Rhodamine B in water [13]. Flower-like Ag NSs have greater surface-enhanced Raman scattering (SERS) enhancement than spherical and disk-like Ag nanoplates do [14]. Those NSs are commonly prepared by different routes such as chemical reduction [15-18], chemical vapor deposition [19-20], hydrothermal reaction [21-23], and pyrolysis [24-26].

Ag nanoparticles (NPs), like gold NPs, possess strong surface plasmon resonance (SPR) and are useful matrixes for enhancement of Raman scattering signals of various analytes and for optothermal conversion (i.e., the transfer of photon energy into thermal energy) [27-34]. Surface enhanced Raman scattering (SERS) up to $10^{6}$ has been demonstrated for the detection of rhodamine $6 \mathrm{G}$ (R6G) when using aggregated Ag NPs having diameters of several tenths of nanometers [34]. These studies have suggested that the SERS signals of R6G are related to the sizes and shapes of the Ag NPs; the

*Address correspondence to this author at the Department of Chemistry, National Taiwan University, Taipei, Taiwan; Tel/Fax: 011-886-2-33661171; E-mail: changht@ntu.edu.tw largest SERS enhancements were achieved when using $\mathrm{Ag}$ NP aggregates having the average sizes ranging from 100 to $250 \mathrm{~nm}$ [27-34]. The optothermal conversion efficiency of NPs is dependent on the plasmon extinction constant of the NPs and the overlap between the extinction band and the emission band of light; i.e., higher optothermal conversion efficiencies are achieved when higher plasmon extinction constants and greater overlap are available [35-37].

Parameters such as the species and concentrations of the metal ions, surfactants, and reducing agents as well as temperature play important roles in determining the sizes and shapes of NSs [15-18]. Polymers such as poly(ethylene glycol) (PEG) and poly(vinylpyrrolidone) (PVP) and surfactants play important roles in controlling the shape, size, and stability of the NFs [26, 38-39]. PEG having a molecular weight $\left(\mathrm{M}_{\mathrm{w}}\right)$ of ca. 20,000 has been used for the synthesis of $\mathrm{Fe}_{3} \mathrm{O}_{4}$ fractal NSs because it prevent the aggregation of $\mathrm{Fe}_{3} \mathrm{O}_{4} \mathrm{NSs}$ and kinetically control the growth rates of the various crystallographic facets of face-centered cubic $\mathrm{Fe}_{3} \mathrm{O}_{4}$ through selective absorption [39]. A solution consisting of PVP and PEG ( $\mathrm{M}_{\mathrm{w}} 400$ and 20,000, respectively) has been used to prepare $\gamma-\mathrm{MnO}_{2} \mathrm{NFs}$ [38]. Solutions containing several stabilizers are useful for the preparation of anisotropic metal NSs [1-2,38-40]. For instance, hyperbranched CdTe and CdSe nanocrystals have been synthesized in the presence of tri-n-octylphosphine (TOPO) and alkylphosphonic acids [1]. Solutions containing two stabilizers are useful for preparation of Ag NSs; e.g., sodium citrate/PVP and PEG/PVP pairs have been used for preparations of Ag nanoprisms and nanorods, respectively $[2,40]$.

In this study, we prepared and characterized $\mathrm{Ag} \mathrm{NFs}$ (rose-shaped), as well as spike- and snowflake-shaped $\mathrm{Ag}$ NSs and tested their potential for use as SERS matrices. Using solutions containing various amounts of PEG $\left(\mathrm{M}_{\mathrm{w}}=\mathrm{ca}\right.$. $8000)$, sodium acetate ( $\mathrm{NaAc}$ ), and sodium citrate, we developed a one-pot synthetic strategy for the preparation of AgNFs, as well as spike- and snowflake-shaped Ag NSs from $\mathrm{Ag}$ ions in aqueous solution. PEG and sodium citrate are common reducing and capping agents for preparing NSs 
[38-40]. NaAc, which is useful for preventing particle agglomeration through electrostatic stabilization, possibly plays a role in assisting the ethylene glycol-mediated reduction of $\mathrm{FeCl}_{3}$ to $\mathrm{Fe}_{3} \mathrm{O}_{4}$ [41-43].

\section{MATERIALS AND METHODOLOGY}

Chemicals and Instruments. Silver nitrate, sodium acetate $(\mathrm{NaAc}), \quad L$-ascorbic acid, sodium citrate and poly(ethylene glycol) $\left(\mathrm{M}_{\mathrm{W}} \sim 8000\right)$ were purchased from Acros (Geel, Belgium) and used as received. A double-beam UV-Vis spectrophotometer (Cintra 10e) obtained from GBC (Victoria, Australia) was used to measure the absorbance of the solutions containing Ag NFs. Transmission electron microscope (TEM) and high-resolution TEM (HR-TEM) images were recorded using H-7100 TEM (Hitachi, Tokyo, Japan) and Tena G2 HR-TEM (Fei, Amsterdam, Netherlands) instruments, respectively. Energy-dispersive X-ray (EDX) spectroscope measurements were obtained using the HR-TEM microscope. Images of thus-prepared Ag NFs were recorded using a Hitachi S-2400 (Hitachi, Tokyo, Japan) scanning electron microscope (SEM). An X-ray powder diffraction instrument (PANalytical) from $X^{\prime}$ Pert PRO (Almelo, Netherlands) was employed to measure the crystal facets of the thus-prepared Ag NFs. A scanning probe microscope (NS3a controller with D3100 stage) from NT-MDT (Moscow, Russia) was used to measure the electronic conductivity of the thus-prepared Ag NFs. The surface area of the thus-prepared Ag NFs was measured at 77K using a Micromeritics ASAP 2010 analyzer (Micromeritics Inc., USA). A continuous diode laser (LD1187, Onset, Taipei, Taiwan) having an output of $1.2 \mathrm{~W}$ was used for determining the optothermal conversion properties of the thus-prepared $\mathrm{Ag}$ NFs, as well as those of the spike- and snowflake-shaped Ag NSs. Raman experiments were performed on a Nicolet Almega dispersive Raman Spectrometer (Thermo Electron Corporation, Waltham, MA) with excitation at $780 \mathrm{~nm}, 100$ $\mathrm{mW}$ of power, and an integration time of $60 \mathrm{~s}$.

Synthesis of Ag NSs. For preparation of Ag NFs, we prepared a typical stabilizing solution by adding $\mathrm{NaAc}(4.5$ $\mathrm{g}, 0.055 \mathrm{~mol})$ and PEG $(1.25 \mathrm{~g}, 0.156 \mathrm{mmol})$ to $5 \mathrm{mM}$ sodium citrate solution $(50 \mathrm{~mL})$. A portion $(1 \mathrm{~mL})$ of this stabilizing solution was mixed with $0.589 \mathrm{mM} \mathrm{Ag}$ ions $(4 \mathrm{~mL})$, distilled/deionized $\mathrm{H}_{2} \mathrm{O}(4 \mathrm{~mL})$, and $0.0788 \mathrm{M}$ ascorbic acid $(1 \mathrm{~mL})$. After incubation for $10 \mathrm{~min}$, the color of the mixture changed to blue, indicating the formation of Ag NSs. Unless noted otherwise, the solution then was subjected to two centrifuge/wash cycles [10,000 rpm for $10 \mathrm{~min}$; washing with 5 $\mathrm{mM}$ sodium citrate $(1 \mathrm{~mL})]$. Finally, the pellet was redispersed in $5 \mathrm{mM}$ sodium citrate solution $(5 \mathrm{~mL})$. Preparations of different shapes of Ag NSs were conducted through similar processes, besides the amounts of three stabilizers are different (detailed compositions see the Result and discussion).

SERS Measurements. Before conducting SERS measurements, we subjected all the thus-prepared Ag NSs to five centrifuge/wash cycles to remove as much of the three stabilizes as possible and to concentrate them. For SERS measurements, we mixed $200 \mu \mathrm{L}$ of concentrated (120 times) Ag NFs or snowflake-shaped Ag NSs with $10^{-7} \mathrm{M}$ R6G (1 mL), $10 \mathrm{mM}$ phosphate $(\mathrm{pH} 12.0,1 \mathrm{~mL})$, and distilled/deionized $\mathrm{H}_{2} \mathrm{O}(7.8 \mathrm{~mL})$, to give a final volume of $10 \mathrm{~mL}$. The result- ing solutions were then incubated for $1 \mathrm{~h}$ to allow the R6G molecules to adsorb onto the surface of the Ag NSs. The Ag NFs and snowflake-shaped Ag NSs were collected separately through centrifugation (10,000 rpm for $10 \mathrm{~min})$; each pellet was then placed onto a glass surface with a sample spot of $50 \mu \mathrm{m}$. In the SERS measurements, the samples were irradiated with light from a $100-\mathrm{mW}$ laser $(780 \mathrm{~nm})$ having a spot size of $1 \mu \mathrm{m}$. The collection time for each SERS spectrum was $60 \mathrm{~s}$.

\section{RESULTS AND DISCUSSION}

Fig. (1a-e) display the transmission electron microscope (TEM), high-resolution TEM (HR-TEM), and scanning electron microscope (SEM) images of the as-prepared roseshaped Ag NSs, also called Ag NFs. The Ag ions had been reduced by ascorbate and the resultant Ag NSs stabilized by the three additives (PEG, sodium citrate, and NaAc). We did not observe the formation of any Ag NSs in the absence of ascorbate, supporting its role as a reducing agent. From the TEM images, we estimate that the Ag NFs had diameters ranging from 800 to $1300 \mathrm{~nm}$, each with a flower bud ranging in the size from 120 to $150 \mathrm{~nm}$ and several petals having lengths from 550 to $650 \mathrm{~nm}$, widths from 200 to $500 \mathrm{~nm}$, and thicknesses from 50 to $100 \mathrm{~nm}$. Most (>90\%) of the Ag NSs are Ag NFs, which was estimated from the TEM image. The rest Ag NSs are spherical and irregular shapes. The HRTEM image in Fig. (1c) depicts clearly that some small Ag NSs (black spheres; $9.1 \pm 1.7 \mathrm{~nm}$ ) and holes $(4.7 \pm 1.1 \mathrm{~nm})$ existed on the surfaces of the Ag NFs, indicating that their surfaces were rough and porous. A selected-area electron diffraction pattern [SAED; Fig. (1f)] indicated the presence of (200) $(d=$ ca. $1.4 \AA)$ and $(422)(d=$ ca. $0.8 \AA)$ crystal facets, suggesting that the as-prepared Ag NFs were singlecrystalline and had face-centered cubic (fcc) structures. Xray diffraction (XRD) and energy dispersed X-ray (EDX) spectra [Fig. (S1a,b), respectively] confirmed that the NFs consisted of $\mathrm{Ag}$ atoms [5,44-45]. The Ag NFs exhibited strong extinction coefficients in the visible-IR region [from 400 to $1200 \mathrm{~nm}$; Fig. (S1c)] as a result of both strong absorption and scattering [46-47]. The as-prepared Ag NFs were slightly hydrophobic, as evidenced by the fact that their contact angles for water were ca. $97.6 \pm 2.2^{\circ}$; in comparison, the surfaces of bulk Ag materials are highly hydrophilic, with contact angles below $10^{\circ}$ [48-49]. The relatively hydrophobic surfaces of the Ag NFs result from the adsorption of the stabilizers onto their surfaces. The BET specific surface area of the Ag NFs was ca. $15.6 \mathrm{~m}^{2} \mathrm{~g}^{-1}$.

We conducted scanning probe microscope (SPM) measurements to obtain a typical current-voltage (I-V) curve of the thus-prepared Ag NFs (Fig. 2a). Prior to the SPM measurement, we subjected the as-prepared Ag NFs to five centrifuge/wash cycles to clean their surfaces [50-52]. When we applied potentials over the range from -300 to $+300 \mathrm{mV}$ at a scan rate at $0.3 \mathrm{~Hz}$ at room temperature, the two limits of the measuring currents were $\pm 152 \mathrm{nA}$. The SPM image in Fig. (2b) depicts a beautifully integrated Ag NF, highlighted by the box. When the applied voltages were set at 200, 300, 400 , and $1000 \mathrm{mV}$, the feedback DC sample bias values were $-10,-15,-20$, and $-50 \mathrm{mV}$, respectively, indicating that the thus-prepared Ag NFs were conductive. From the I-V curve, the conductivity ( $\rho$ ) of the thus-prepared Ag NFs could be estimated using Equation (1) [50-52]: 


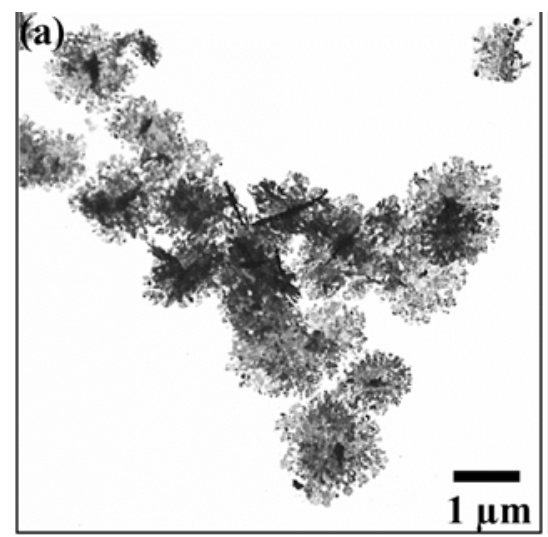

(b)
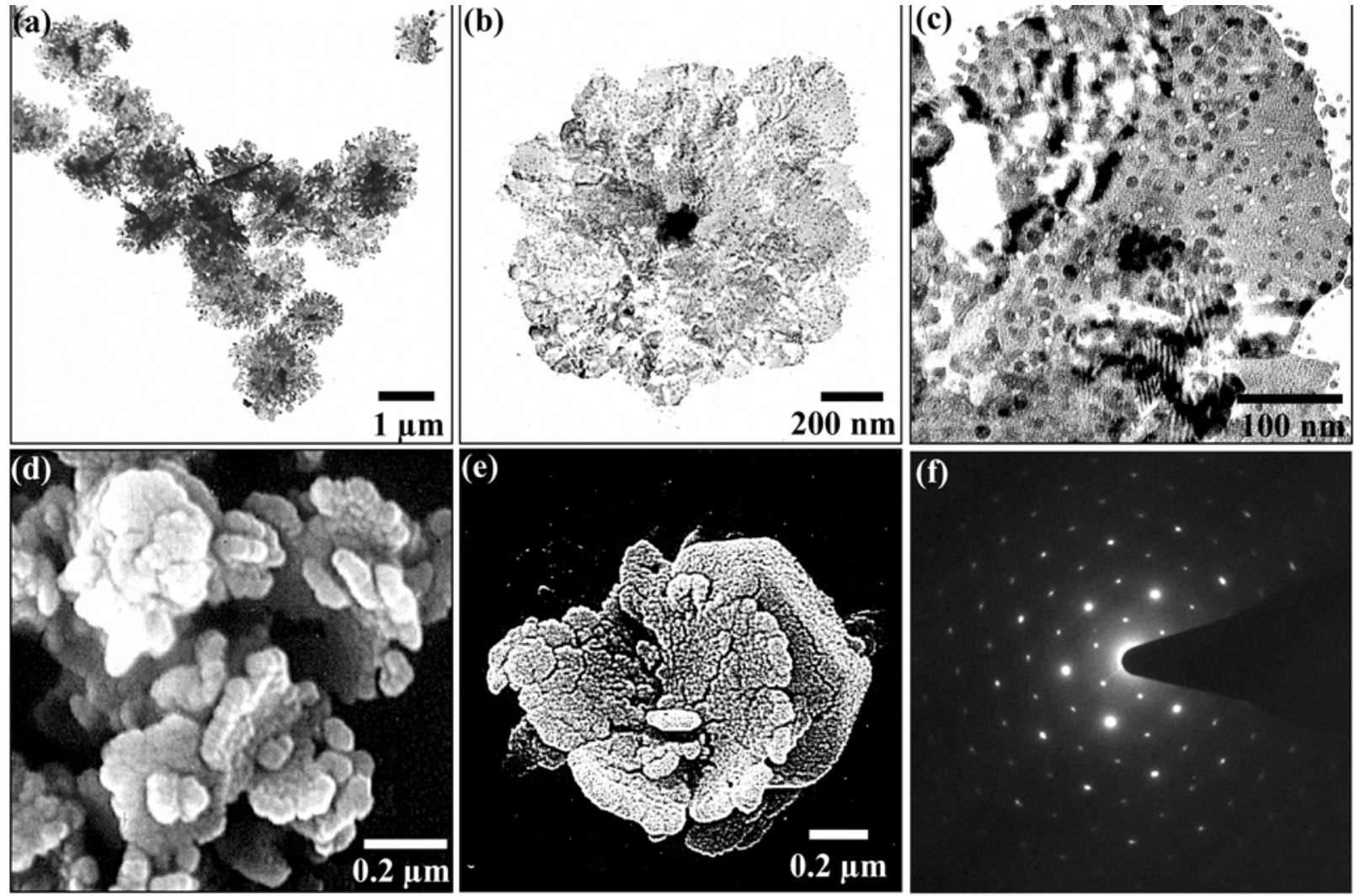

Fig. (1). TEM images of (a) Ag NFs and (b) a representative Ag NF. (c) High-magnification HR-TEM image of an Ag NF exhibited in (b). SEM images of (d) Ag NFs and (e) a representative Ag NF. (f) Selected-area electron diffraction pattern of an Ag NF displayed in (b).

$\rho=\mathrm{L} / \mathrm{AR}$

where $\mathrm{L}$ is the thickness of the specimen, $\mathrm{A}$ is the area between the SPM probe and the specimen (which was $7.85 \times$ $10^{-11} \mathrm{~cm}^{2}$, provided that the SPM probe radius was $50 \mathrm{~nm}$ ), and $\mathrm{R}$ is the electrical resistance of the specimen. From the inverse linear slope of the $\mathrm{I}-\mathrm{V}$ curve (amplitude sensitivity $=$ $10 \mathrm{nAV}^{-1}$ ), we calculated $\mathrm{R}$ to be $0.0217 \Omega$. From SPM analyses, we estimated the thickness (L) of the Ag NFs to be
28-39 nm (average: ca. $33.5 \mathrm{~nm}$ ). By incorporating these values into Equation 1, we calculated the conductivity of the thus-prepared $\mathrm{Ag} \mathrm{NFs}$ to be $1.97 \times 10^{6} \mathrm{~S} \mathrm{~cm}^{-1}$; i.e., our asprepared flower-shaped Ag NSs were highly conductive. For comparison, we note that the conductivity of $\mathrm{Ag}$ bulk materials is $6.30 \times 10^{7} \mathrm{~S} \mathrm{~m}^{-1}$. The lower conductivity of thusprepared Ag NFs is likely due to adsorption of surfactants and defected structures.
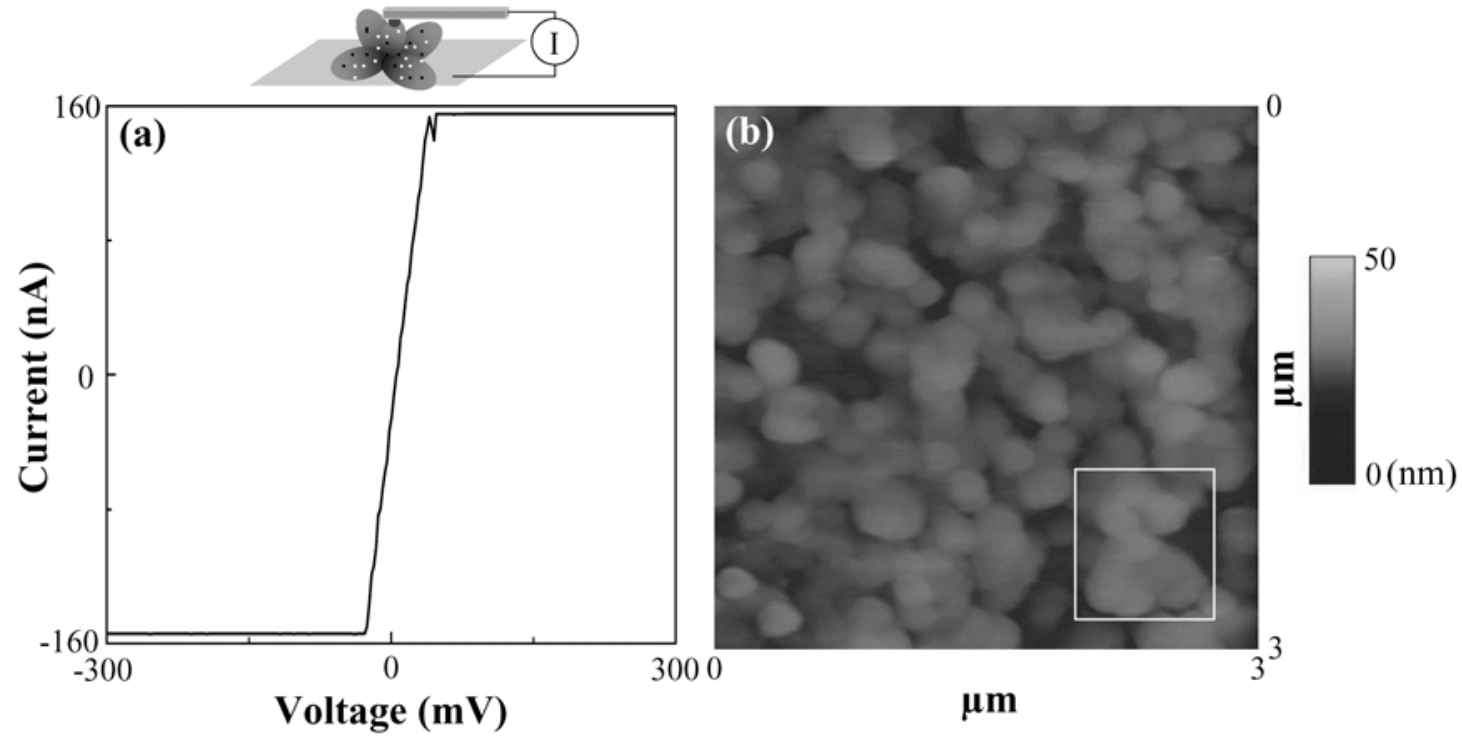

Fig. (2). (a) $I-V$ characteristic curve and (b) SPM image of Ag NFs. The box highlighted in (b) clearly exhibits a Ag NF. 


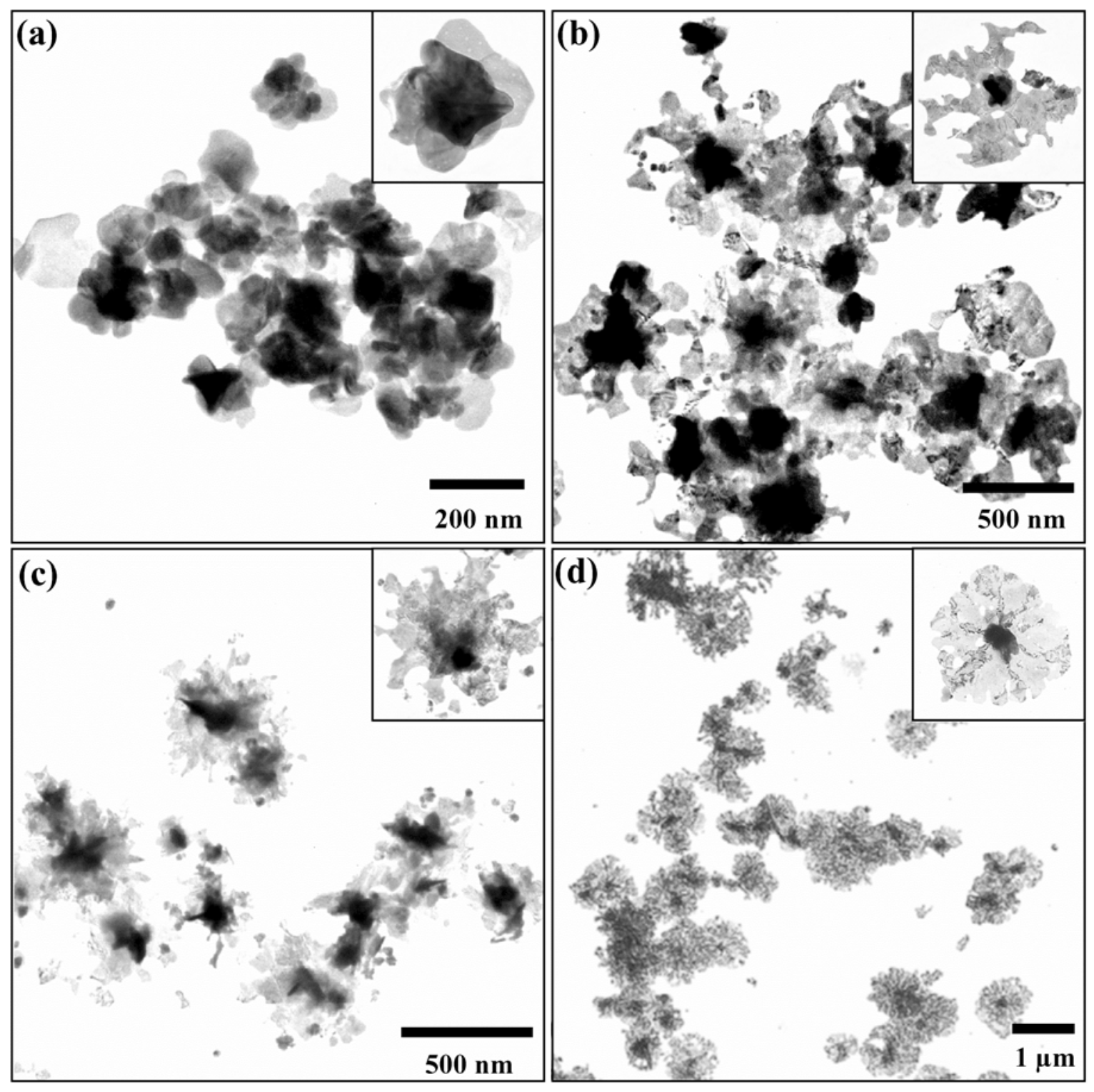

Fig. (3). TEM images of Ag NSs recorded over the course of the reaction: (a) 1, (b) 2.5, (c) 5, and (d) 7.5 min. The amplified TEM image in each inset displays a representative Ag NS. Preparation conditions were similar to those described in Fig. (1).

To explore the crystal growth of the Ag NFs, we stopped the reactions at various points by quickly separating the NSs from the solutions through centrifugation at $10,000 \mathrm{rpm}$ for 1 min $[1,53]$. The HRTEM images in Fig. (3a-d) exhibit the NSs after reaction periods of $1,2.5,5$, and $7.5 \mathrm{~min}$, respectively $[1,21]$. When the reaction time was $1 \mathrm{~min}$, small petals (length: 60-80 nm; width: 50-80 nm) were formed (Fig. 3a). Because many of those petals lacked buds, we suggest that initially the $\mathrm{Ag}$ atoms formed single petals, upon which other petals formed subsequently at their termini as a result of deposition of $\mathrm{Ag}$ atoms. This process repeated until the desired number of petals formed. As a result of more Ag atoms being deposited, the bud became more apparent in each $\mathrm{Ag}$ NS. The petals gradually grew in different directions to form larger petals that had lengths of $400-500 \mathrm{~nm}$ and widths of $150-300 \mathrm{~nm}$ when the reaction time approached $2.5 \mathrm{~min}$, as exhibited in Fig. (3b). It is interesting to note that several petals were either separated or connected with adjacent ones for each NS at this stage. In the connection area, some large open areas are apparent. When the reaction times were over 5-7.5 min, Fig. (3c) indicates that the petals overlapped and the large open areas disappeared as a result of the further deposition of $\mathrm{Ag}$ atoms. When the reaction time approached $10 \mathrm{~min}, \mathrm{Ag}$ NFs were formed. We note that the flower buds also grew in diameter from ca. 50 to ca. $150 \mathrm{~nm}$ as the reaction time increased from 1 to $10 \mathrm{~min}$ as a result of the gradual deposition of $\mathrm{Ag}$ atoms. The time dependence visible-IR spectra for the as-prepared Ag NSs were shown in Fig. (S2). Since scattering has greater influence on the absorption spectra when the size of $\mathrm{Ag}$ NSs becomes larger, the extinction values are not simply related to the concentrations and size of AgNSs.

Next, we investigated the roles that the three stabilizers played during the synthesis of the Ag NFs. First, we prepared Ag NSs in the presence of the individual capping agents at different concentrations, with the results displayed in Fig. (4). When using 0.312 M PEG as the stabilizer, we 
prepared only snowflake-shaped Ag NSs (Fig. 4c). The major petals for snowflake-shaped NSs had lengths of ca. 800$1000 \mathrm{~nm}$ and widths of 200-250 nm. On the large petals' surfaces, some small dendrimer-shaped petals $(140-280 \mathrm{~nm}$ long and 80-150 nm wide) were also apparent. At 0.078, 0.156, and 0.624 M PEG, dendrimer-shaped Ag NSs were prepared. When sodium citrate $(0.125 \sim 1 \mathrm{mM})$ was used as the sole stabilizer, spike-shaped Ag NSs (330-430 nm long) were synthesized (Fig. 4g). It is apparent that more Ag atoms deposited on the central (dark) part of each spike-shaped Ag NS. In contrast, Fig. (4k) indicates that only spherical Ag NSs $(117 \pm 32 \mathrm{~nm})$ were synthesized when NaAc $(0.055 \sim 0.11 \mathrm{M})$ was used as the stabilizer. In addition, starshaped Ag NSs were formed at lower concentration of NaAc (0.0275 M) (Fig. 4i), while spherical- and prism-like Ag NPs were synthesized at higher concentration of $\mathrm{NaAc}(0.22 \mathrm{M})$ (Fig. 4l). The dispersed Ag NSs exhibited in these TEM images clearly indicate that the three capping agents are all effective stabilizers, but they play different roles in controlling the formation of the variously shaped and sized Ag NSs. Next, we prepared Ag NSs in stabilizer pair systems; typical mixtures included $0.312 \mathrm{M}$ PEG, $0.5 \mathrm{mM}$ sodium citrate and $0.11 \mathrm{M} \mathrm{NaAc}$. Unlike those NSs formed in the triplestabilizer and single-stabilizer systems, in all of these cases we synthesized only irregular spherical-shaped Ag NPs in different sizes.
We also investigated the preparation of Ag NSs in different concentrations of the triple-stabilizer systems; half $(0.5 X)$, twice $(2 X)$, and four times $(4 X)$ the original concentrations (1X) with respect to the individual stabilizers, as described in Fig. (1). From the $0.5 \mathrm{X}$ triple-stabilizer system, we prepared spike-shaped Ag NSs, as exhibited in the TEM images in Fig. (S3a). The TEM images in Fig. (S3b,c) indicate that Ag NFs and spherical Ag NPs (120-260 nm diameters) were prepared in the $2 \mathrm{X}$ and $4 \mathrm{X}$ triple-stabilizer systems, respectively. We summarize the impact of stabilizers in Scheme 1.

These results indicate that the concentration and nature of the three stabilizers plays an important role in determining the morphologies of the Ag NSs, mainly through controlling the kinetics of their growth. At low concentrations, very few Ag seeds formed initially as a result of only a few stabilizer molecules existing in each seed; thus, the seeds' growth became dominant, leading to the formation of snowflakeshaped Ag NSs [54]. At high concentrations, more Ag seeds formed initially, leading to the formation of small spherical Ag NPs. In addition, the different stabilities and rates of reduction (by ascorbic acid) of the various Ag ion complexes at each concentration of the three stabilizers were also contributors for determining their morphologies [54].
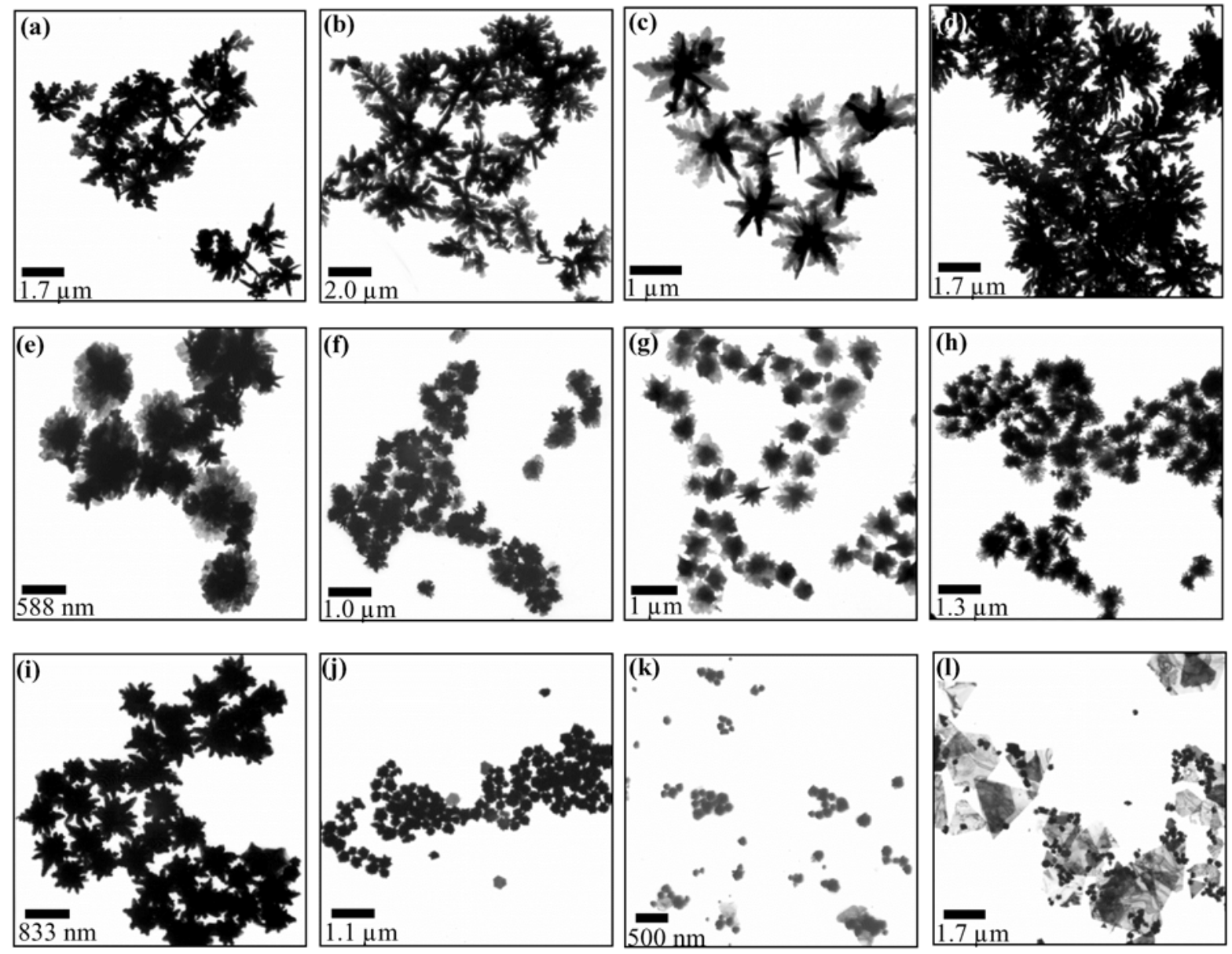

Fig. (4). TEM images of Ag NSs prepared in a single stabilizer at different concentrations. PEG: (a) 0.078 M, (b) $0.156 \mathrm{M}$, (c) $0.312 \mathrm{M}$, and (d) 0.624M. Sodium citrate: (e) $0.125 \mathrm{mM}$, (f) $0.25 \mathrm{mM}$, (g) $0.5 \mathrm{mM}$, and (h) $1 \mathrm{mM}$. NaAc: (i) $0.0275 \mathrm{M},(\mathbf{j}) 0.055 \mathrm{M}$, (k) $0.11 \mathrm{M}$, and (l) $0.22 \mathrm{M}$. 


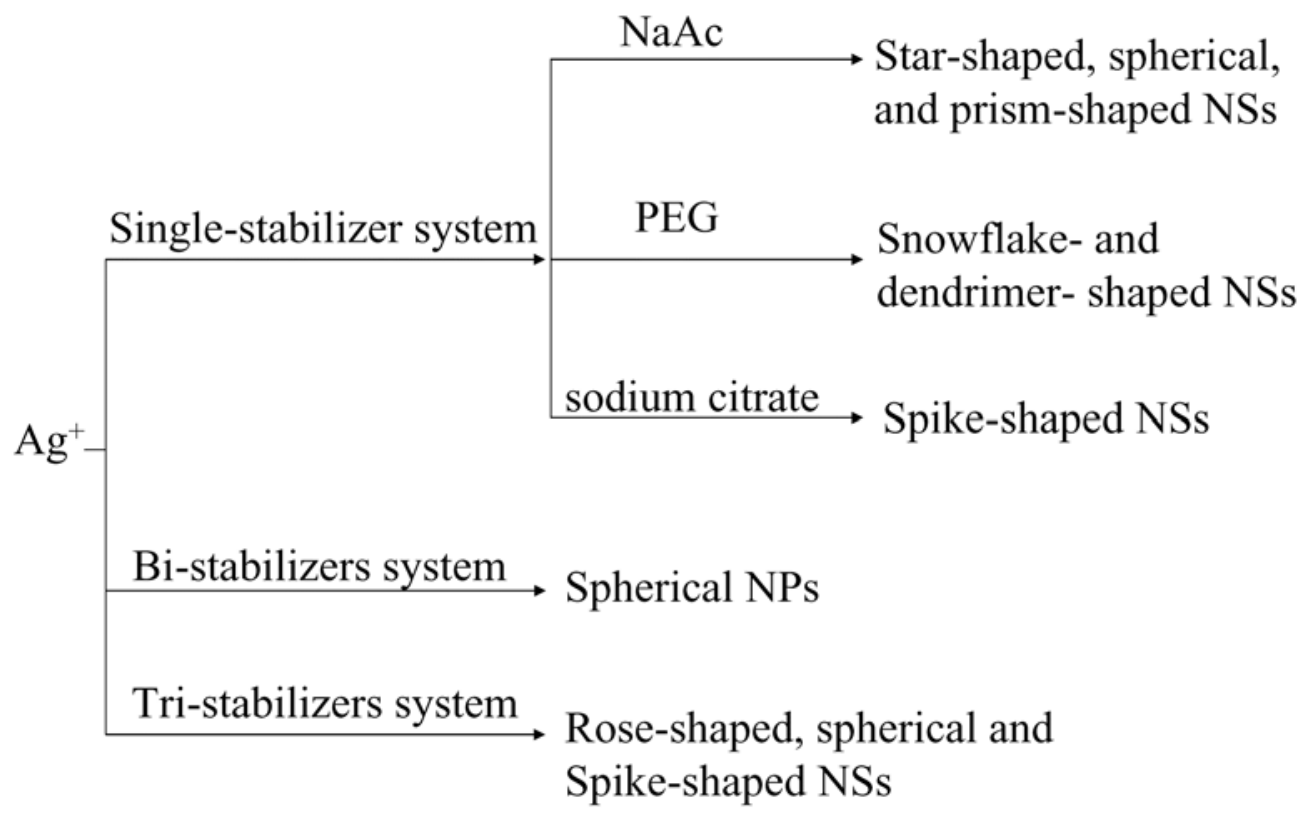

Scheme 1. Impact of capping agents on the formation of different shapes of Ag NSs.

In order to test the possibility of using the as-prepared $\mathrm{Ag}$ NSs for photothermal therapy [35-37], we conducted optothermal conversion measurements. Because of their strong extinction in the visible-IR region (400-1200 nm), we measured the optothermal conversion properties of the thusprepared Ag NFs, as well as those of the spike- and snowflake-shaped Ag NSs, using irradiation (808 $\mathrm{nm}$ ) from a continuous-wavelength diode laser having an output of $1.2 \mathrm{~W}$. After 3 min of irradiation, the temperature of a $0.1-\mathrm{mL}$ solution of Ag NFs (50X; i.e., concentrated 50-times) increased by $23.03 \pm 0.21{ }^{\circ} \mathrm{C}$ from the initial temperature $\left(22^{\circ} \mathrm{C}\right)$. In the absence of the $\mathrm{Ag} \mathrm{NFs}$, the temperature of a distilled/deionized $\mathrm{H}_{2} \mathrm{O}$ solution $(0.1 \mathrm{~mL})$ rose by $9.63 \pm 0.23$ ${ }^{\circ} \mathrm{C}$. Thus, the presence of the thus-prepared Ag NFs raised the temperature by an additional $13.40 \pm 0.31{ }^{\circ} \mathrm{C}$, i.e., efficient energy transfer occurred from NIR photons into heat [35-37]. The other snowflake- and spike-shaped Ag NSs also exhibited high extinctions in the NIR region, as depicted in Fig. (S4); thus, we expected that they could provide large optothermal conversions under NIR irradiation. The "real" raised temperatures of the snowflake- and spike-shaped $\mathrm{Ag}$ NS solutions were $4.4 \pm 0.24$ and $9.34 \pm 0.31{ }^{\circ} \mathrm{C}$, respectively. Our results suggest that the optothermal conversion efficiency of the Ag NFs was higher than those of the other two Ag NS morphologies, mainly because of their stronger absorption in the NIR region [38-40]. However, the AgNSs must be further functionalized with biomolecules before they can be practical.

Next, we applied the thus-prepared Ag NSs to SERS measurements of R6G. The TEM images suggested that the snowflake-shaped Ag NSs might be more suitable than the Ag NFs for SERS, because of their petal sizes $(80-280 \mathrm{~nm})$ and highly anisotropic geometries, which we expected would greatly enhance the local field intensity near the tips. We note that hot spots of large field enhancements near the tips are ideal for SERS experiments, and that NSs having sizes of 100-200 nm can induce large SERS signals of molecules
[27-34]. When using the Ag NFs, we obtained only the SERS signals at $1507 \mathrm{~cm}^{-1}$ (the strongest peak) of R6G at concentrations greater than $10 \mathrm{nM}$. Fig. (5a) displays the SERS signals for $100 \mathrm{nM}$ R6G obtained using the snowflake-shaped Ag NSs; we observe six representative peaks for R6G at 1186, 1310, 1358, 1507, 1594, and $1636 \mathrm{~cm}^{-1}$ [27-34]. Of these signals, the peaks at 1358, 1507, and 1636 $\mathrm{cm}^{-1}$ have been assigned as representing aromatic $\mathrm{C}-\mathrm{C}$ bond stretching vibration modes [34]. Fig. (5b) indicates that the six representative peaks for the SERS signals of R6G are still assignable at a concentration of $10 \mathrm{nM}$. For comparison, Fig. (5c) depicts the SERS signals for 0.01 M R6G in the absence of the snowflake-shaped Ag NS matrix. From an analysis of the SERS signal at $1507 \mathrm{~cm}^{-1}$, we estimated the enhancement factor $(\mathrm{G})$ of the intensities in the presence and absence of the snowflake-shaped Ag NSs to be ca. $2.4 \times 10^{6}$, according to the modified equation originally proposed by Cai et al. and Green et al. [55-57].

\section{CONCLUSION}

We have demonstrated a one-pot synthetic strategy for the preparation of differently sized and shaped Ag NSs through the reduction of $\mathrm{Ag}$ ions by ascorbate in the presence of PEG, sodium citrate, and NaAc. By carefully controlling the nature and the concentration of the capping agents, this simple approach allows the preparation of snowflake- and spike-shaped Ag NSs, and Ag NFs. The thusprepared Ag NFs were hydrophobic and possessed high electrical conductivities, strong extinction coefficients in the visible-IR region (400-1200 nm), and high degrees of optothermal conversion. Furthermore, snowflake-shaped $\mathrm{Ag}$ NSs led to an amplification of the SERS signals of R6G of at least $2.4 \times 10^{6}$ times.

\section{ACKNOWLEDGEMENTS}

This study was supported by the National Science Council of Taiwan under contract numbers NSC 94-2113-M-002008 and NSC 95-2113-M-002-026-MY3. T.-C.C. is grateful 


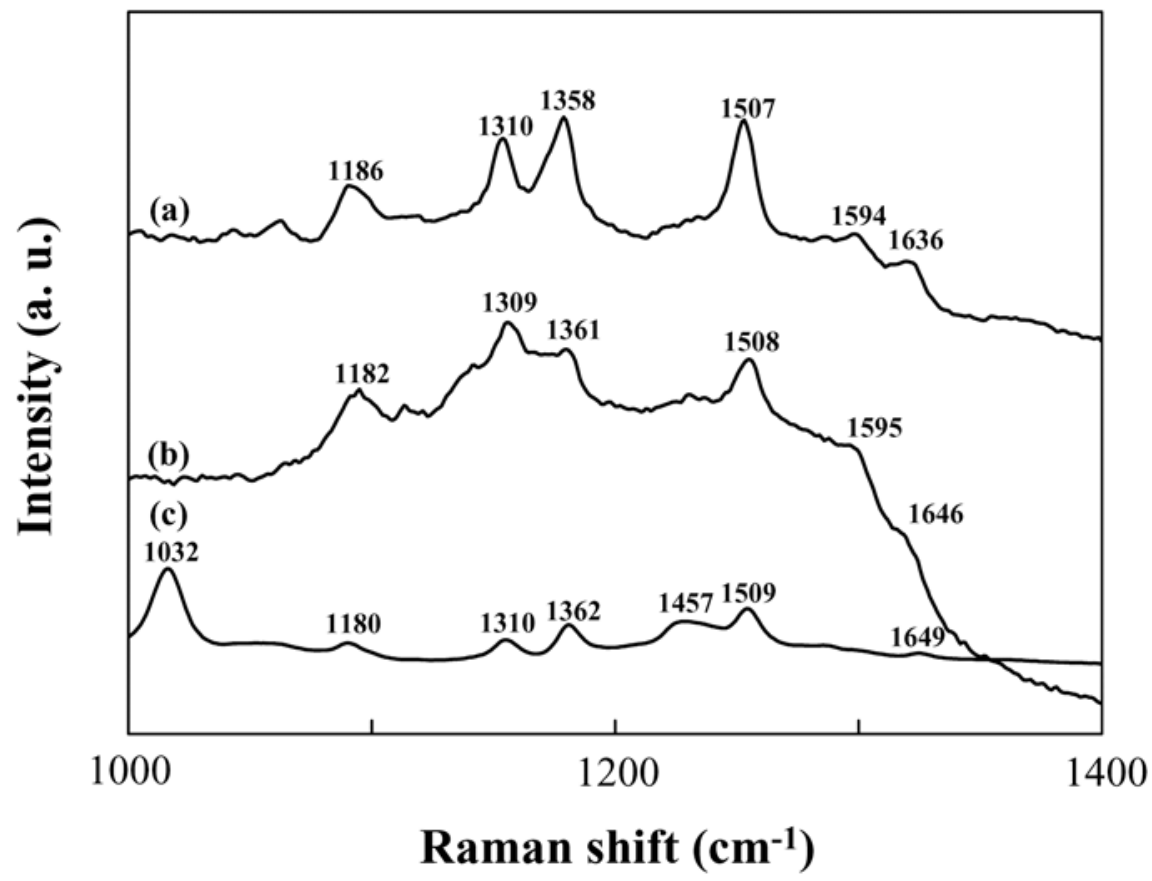

Fig. (5). SERS spectra of (a) $100 \mathrm{nM}$ and (b) $10 \mathrm{nM}$ R6G molecules recorded in the presence of snowflake-shaped Ag NSs, and (c) $10 \mathrm{mM}$ R6G in the absence of Ag snowflakes.

to the National Science Council for awarding his postdoctoral fellowship in the Department of Chemistry, National Taiwan University, under contract number NSC 95-2811-M002-051.

\section{REFERENCES}

[1] Kanaras, A.G.; Sönnichsen, C.; Liu, H.; Alivisatos, A.P. Nano Lett., 2005, 5, 2164.

[2] Métraux, G.S.; Mirkin, C.A. Adv. Mater., 2005, 17, 412.

[3] Chen, S.; Fan, Z.; Carroll, D.L. J. Phys. Chem. B, 2002, 106, 10777.

[4] Hu, J.; Zhang, Y.; Liu, B.; Liu, J.; Zhou, H.; Xu, Y.; Jiang, Y.; Yang, Z.; Tian, Z. Q. J. Am. Chem. Soc., 2004, 126, 9470.

[5] Jin, R.; Cao, Y.W.; Mirkin, C.A.; Kelly, K.L.; Schatz, G.C.; Zheng, J.G. Science, 2001, 294, 1901.

[6] Tang, Z.; Kotov, N.A.; Giersig, M. Science, 2002, 297, 237.

[7] Murray, C.B.; Norris, D.J.; Bawendi, M.G. J. Am. Chem. Soc., 1993, 115, 8706.

[8] Yin, Y.; Rioux, R.M.; Erdonmez, C.K.; Hughes, S.; Somorjai, G.A.; Alivisatos, A.P. Science, 2004, 304, 711.

[9] Fang, X.S.; Ye, C.H.; Xie, T.; Wang, Z.Y.; Zhao, J.W.; Zhang, L.D. Appl. Phys. Lett., 2006, 88, 013101.

[10] Liu, B.D.; Bando, Y.; Tang, C.C.; Golberg, D.; Xie, R.G.; Sekiguchi, T. Appl. Phys. Lett., 2005, 86, 083107.

[11] Chen, A.; Peng, X.; Koczkur, K.; Miller, B. Chem. Commun., 2004, 17, 1964.

[12] Agrios, A.G.; Cesar, I.; Comte, P.; Nazeeruddin, M.K.; Grätzel, M. Chem. Mater., 2006, 18, 5395 .

[13] Wu, J.M.; Qi, B.; J. Phys. Chem. C, 2007, 111, 666.

[14] Lu, L.; Kobayashi, A.; Tawa, K.; Ozaki, Y. Chem. Mater., 2006, $18,4894$.

[15] Ni, X.; Zhao, Q.; Zheng, H.; Li, B.; Song, J.; Zhang, D.; Zhang, X. Eur. J. Inorg. Chem., 2005, 2005, 4788.

[16] Fang, X.S.; Ye, C.H.; Zhang, L.D.; Zhang, J.X.; Zhao, J.W.; Yan, P. Small, 2005, 1,422 .

[17] Pan, A.; Yu, R.; Xie, S.; Zhang, Z.; Jin, C.; Zou, B. J. Cryst. Growth, 2005, 282, 165.

[18] Wen, X.; Xie, Y.T.; Mak, W.C.; Cheung, K.Y.; Li, X.Y.; Renneberg, R.; Yang, S. Langmuir, 2006, 22, 4836.

[19] Hao, Y.; Meng, G.; Zhou, Y.; Kong, M.; Wei, Q.; Ye, M.; Zhang, L. Nanotechnology, 2006, 17, 5006.
[20] Xu, F.; Yu, K.; Li, G.; Li, Q.; Zhu, Z. Nanotechnology, 2006, 17, 2855.

[21] Sun, X.H.; Lam, S.; Sham, T.K.; Heigl, F.; Jürgensen, A.; Wong, N.B. J. Phys. Chem. B, 2005, 109, 3120.

[22] Yan, C.; Xue, D.; Zou, L.; Yan. X.; Wang, W. J. Cryst. Growth, 2005, 282, 448 .

[23] Zhu, H.; Yang, D.; Zhang, H. Mater Lett., 2006, 60, 2686.

[24] Narayanaswamy, A.; Xu, H.; Pradhan, N.; Kim, M.; Peng, X. J. Am. Chem. Soc., 2006, 128, 10310.

[25] Narayanaswamy, A.; Xu, H.; Pradhan, N.; Peng, X. Angew. Chem. Int. Ed., 2006, 45, 5361 .

[26] Chen, J.; Herricks, T.; Geissler, M.; Xia, Y. J. Am. Chem. Soc., 2004, 126, 10854.

[27] Itoh, T.; Biju, V.; Ishikawa, M.; Kikkawa, Y.; Hashimoto, K.; Ikehata, A.; Ozaki, Y. J. Chem. Phys., 2006, 124, 134708.

[28] Lin, W.C.; Yang, M.C. Macromol. Rapid Commun., 2005, 26, 1942.

[29] Li, X.; Wang, Y.; Jia, H.; Song, W.; Zhao, B. J. Raman Spectrosc., 2005, 36, 635 .

[30] Lu, L.; Eychmuller, A.; Kobayashi, A.; Hirano, Y.; Yoshida, K.; Kikkawa, Y.; Tawa, K.; Ozaki, Y. Langmuir, 2006, 22, 2605.

[31] Xu, H.; Bjerneld, E.J.; Käll, M.; Börjesson, L. Phys. Rev. Lett., 1999, 83, 4357.

[32] Michaels, A.M.; Jiang, J.; Brus, L. J. Phys. Chem. B, 2000, 104 11965.

[33] Moyer, P.J.; Schmidt, J.; Eng, L.M.; Meixner, A.J.; Sandmann, G.W.; Dietz, H.; Plieth, W. J. Am. Chem. Soc., 2000, 122, 5409.

[34] Hildebrandt, P.; Stockburger, M. J. Phys. Chem., 1984, 88, 5935.

[35] Richardson, H.H.; Hickman, Z.N.; Govorov, A.O.; Thomas, A.C.; Zhang, W.; Kordesch, M.E. Nano Lett., 2006, 6, 783.

[36] Chou, C.H.; Chen, C.D.; Wang, C.R.C. J. Phys. Chem. B, 2005, $109,11135$.

[37] Hirsch, L.R.; Stafford, R.J.; Bankson, J.A.; Sershen, S.R.; Rivera, B.; Price, R.E.; Hazle, J.D.; Halas, N.J.; West, J.L. Proc. Natl. Acad. Sci. U.S.A., 2003, 100, 13549.

[38] Wu, C.; Xie, Y.; Wang, D.; Yang, J.; Li, T.; J. Phys. Chem. B, 2003, 107, 13583.

[39] Zou, G.; Xiong, K.; Jiang, C.; Li, H.; Li, T.; Du, J.; Qian, Y. J. Phys. Chem. B, 2005, 109, 18356.

[40] Chen, H.; Gao, Y.; Zhang, H.; Liu, L.; Yu, H.; Tian, H.; Xie, S.; Li, J. J. Phys. Chem. B, 2004, 108, 12038 .

[41] Deng, H.; Li, X.; Peng, Q.; Wang, X.; Chen, J.; Li, Y. Angew. Chem. Int. Ed., 2005, 44, 2782. 
[42] Brown, K.R.; Walter, D.G.; Natan, M.J. Chem. Mater., 2000, 12, 306.

[43] Viau, G.; Toneguzzo, P.; Pierrard, A.; Acher, O.; Fiévet-Vincent, F.; Fiévet, F. Scr. Mater., 2001, 44, 2263.

[44] Hanawalt, J.D.; Rinn, H.W.; Frevel, L.K. Anal. Chem., 1938, 10, 457.

[45] Kirkland, A.I.; Jefferson, D.A.; Duff, D.G.; Edwards, P.P.; Gameson, I.; Johnson, B.F.G.; Smith, D.J. Proc. R. Soc. Lond. A, 1993, 440, 589.

[46] Pang, S.; Kondo, T.; Kawai, T. Chem. Mater., 2005, 17, 3636.

[47] Wang, S.; Xin, H. J. Phys. Chem. B, 2000, 104, 5681.

[48] Osman, M.A.; Keller, B.A. Appl. Surf. Sci., 1996, 99, 261.

[49] Schrader, M.E. J. Phys. Chem., 1974, 78, 87.
[50]

[51]

$[52]$

[54] Libbrecht, K.G. Rep. Prog. Phys., 2005, 68, 855.
[55] Cai, W.B.; Ren, B.; Li, X.Q.; She, C.X.; Liu, F.M.; Cai, X.W.; Tian, Z.Q. Surf. Sci., 1998, 406, 9.

[56] Green, M.; Liu, F.M. J. Phys. Chem. B, 2003, 107, 13015

[57] Orendorff, C.J.; Gole, A.; Sau, T.K.; Murphy, C.J. Anal. Chem., 2005, 77, 3261 . 